\title{
Fast-Scale Instability of Single-Stage Power-Factor-Correction Power Supplies
}

\author{
Xiaoqun Wu, Chi K. Tse, Fellow, IEEE, Octavian Dranga, and Junan Lu
}

\begin{abstract}
This paper describes the fast-scale bifurcation phenomena of a single-stage power-factor-correction (PFC) power supply which is a cost effective design for low-power applications. The circuit employs a cascade configuration of a boost converter and a forward converter, which share an active switch and operate in discontinuous conduction mode, to provide input PFC and tight output regulation. Main results are illustrated by "exact" circuit simulations as well as theoretical analysis based on the use of Jacobians. This work provides a convenient means of predicting stability boundaries which can facilitate the selection of practical parameter values for maintaining stable operation.
\end{abstract}

Index Terms-Bifurcation, dc-dc converter, instability, power factor correction (PFC), single-stage converter.

\section{INTRODUCTION}

$\mathbf{T}$ HE single-stage isolated power-factor-correction (PFC) power supply (SSIPP) proposed by Redl et al. [1] is a cost effective design solution for power supplies that are required to provide PFC and tight output regulation in the low-power range. The circuit basically employs a cascade structure consisting of a boost PFC converter and a forward converter for output regulation [1]. Being a single-stage converter, the SSIPP uses one active switch, as shown in Fig. 1, and hence mandatorily operates the PFC stage in discontinuous conduction mode (DCM) in order to allow the duty cycle control to be solely deployed for output regulation since the DCM boost converter can automatically achieve a good power factor without additional control [2], [3]. Furthermore, such an operating mode, as pointed in the Redl et al. proposal [1], avoids a variable voltage stress for the storage capacitor which sits between the two stages. Due to its practical versatility for low-power applications, this circuit has received a great deal of attention in the past decade [4]-[6]. However, previous studies have mainly focused on its steady-state design and control aspects, the detailed dynamical behavior and the stability boundaries have not been thoroughly pursued.

Recently, studies of the dynamical behavior of switching power converter circuits have revealed the possibilities of

\footnotetext{
Manuscript received November 3, 2004; revised April 22, 2005. This work was supported by the Hong Kong Research Grants Council under a competitive earmarked Research Grant (PolyU 5241/03E). This paper was recommended by Associate Editor M. K. Kazimierczuk.

$\mathrm{X} . \mathrm{Wu}$ is with the Department of Electronic and Information Engineering, Hong Kong Polytechnic University, Hong Kong, and also with the School of Mathematics and Statistics, Wuhan University, Hubei 430072, China.

C. K. Tse and O. Dranga are with the Department of Electronic and Information Engineering, Hong Kong Polytechnic University, Hong Kong (e-mail: encktse@polyu.edu.hk).

J. Lu is with the School of Mathematics and Statistics, Wuhan University, Hubei 430072, China.

Digital Object Identifier 10.1109/TCSI.2005.854293
}

various kinds of bifurcation behavior, e.g., in pulsewidth-modulated (PWM) dc-dc converters [7]-[12], current-mode controlled dc-dc converters [13]-[15], thyristor and diode circuits [16], [17], free-running dc-dc converters [18], parallel-connected dc-dc converters [19], [20], general switching circuits [21], [22], etc. For the boost PFC preregulators operating in continuous conduction mode (CCM), it has been found that both fast-scale and slow-scale instabilities are possible [23]-[25]. However, similar problems in the DCM counterparts are known by practitioners but are not systematically explained and studied. Clearly, the slow-scale instability problem may worsen the harmonic distortion of the input current, whereas the fast-scale instability problem may impose higher current stresses on the switching devices. It has been observed that the peak current can increase by $10 \%-20 \%$ when fast-scale instabilities occur in some intervals of the mains cycle [24], [25]. Thus, the study of such instability problems has a practical motivation. See also Banerjee and Verghese [26] and Tse [27] for some surveys of the recent research in this area.

In this paper, we report fast-scale period-doubling bifurcation observed in the complete single-stage PFC power supply, in which both the PFC boost preregulator and the forward output regulator are designed to operate in DCM. In this paper, we will show that, with improper choice of system parameters, the converter can suffer from fast-scale instability for some intervals of time during the line cycle. This is important because line current distortions are often resulted from occasional instabilities that occur in some parts of the line cycle. Computer simulations based upon exact cycle-by-cycle system equations are presented in this paper. The extents to which fast-scale instability may occur are quantitatively measured and the parameters that affect fast-scale instability are identified. Analytical equations and design curves are derived from discrete-time models to facilitate the design of this type of power supplies to avoid fast-scale instability for all times.

\section{SYSTEM DESCRIPTION}

The SSIP converter under study is shown in its original form in Fig. 1 [1]. The front-end boost converter serves as a PFC converter whose output is connected across the storage capacitor $C_{1}$, which in turn serves as the input to a standard forward converter. Moreover, the boost PFC converter and the forward converter share the same active switch $S$, as shown in Fig. 1. Thus, this circuit can be modeled as a cascade connection of a boost converter and a buck converter, which are driven synchronously under one switching PWM signal, as shown in Fig. 2 [3]. The control uses a simple PWM scheme, in which a control voltage 


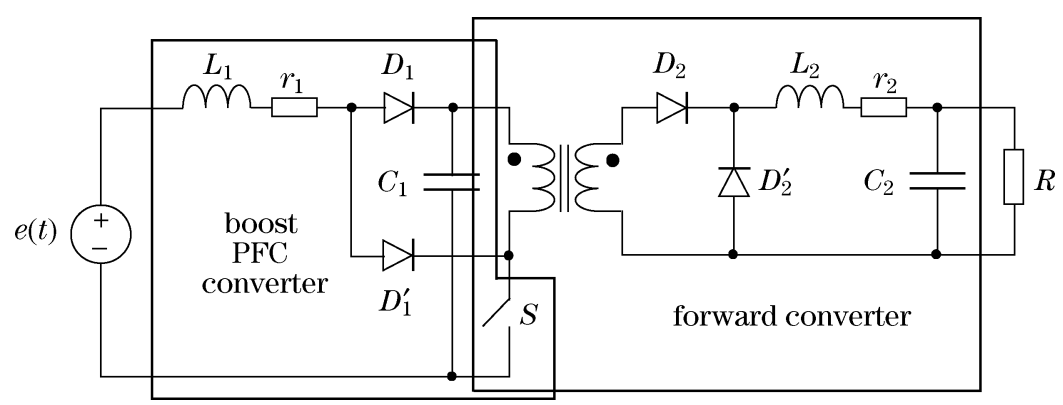

Fig. 1. The SSIPP [1]. This circuit consists of a boost front-end PFC converter and a forward converter. Transformer isolation allows sharing of active switch by the two cascading stages [5], [6]. For brevity, the core reset arrangement is omitted.

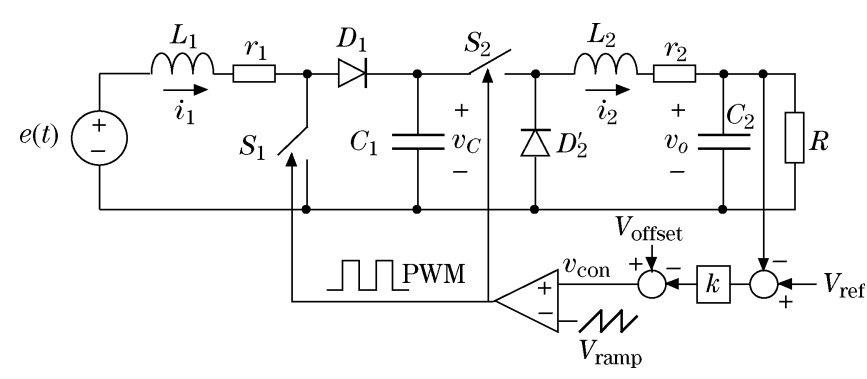

Fig. 2. Equivalent circuit model of the single-stage PFC power supply.

$v_{\text {con }}$ is compared with a sawtooth signal to generate a PWM signal to drive the switches. The sawtooth signal is given by

$$
V_{\text {ramp }}=V_{L}+\left(V_{U}-V_{L}\right) \frac{t \bmod T}{T}
$$

where $V_{L}$ and $V_{U}$ are the lower and upper voltage limits of $V_{\text {ramp }}$, and $T$ is the switching period. The PWM signal is "high" when $v_{\text {con }}>V_{\text {ramp }}$, and is 'low' otherwise. The control voltage $v_{\text {con }}$ is derived from a voltage feedback loop, i.e.,

$$
v_{\text {con }}=V_{\text {offset }}-k\left(v_{o}-V_{\text {ref }}\right)
$$

where $V_{\text {ref }}$ is the reference output voltage, $k$ is the feedback gain, and $V_{\text {offset }}$ is a dc voltage that gives the required steadystate duty cycle, i.e., $V_{\text {offset }}=V_{L}+D\left(V_{U}-V_{L}\right)$.

When both the boost and the buck stages are working in DCM, five switch states are possible during a switching cycle:

State A: $S_{1}$ and $S_{2}$ are on, $D_{1}$ and $D_{2}$ are off;

State B: $S_{1}$ and $S_{2}$ are off, $D_{1}$ and $D_{2}$ are on;

State C: $S_{1}$ and $S_{2}$ are off, $D_{1}$ is on and $D_{2}$ is off;

State D: $S_{1}$ and $S_{2}$ are off, $D_{1}$ is off and $D_{2}$ is on;

State E: $S_{1}$ and $S_{2}$ are off, $D_{1}$ and $D_{2}$ are off.

It is worth noting that the sequence of switch states, in general, takes the order as written above. However, either State C or State D (not both) goes in the middle because exact synchronous switching of the diodes is not possible in practice. For simplicity in our study, we will omit State C, assuming that the buck stage has a relatively larger inductance. Typical current waveforms are illustrated in Fig. 3. Note that the choice between omitting State $\mathrm{C}$ or D must be consistent with the choice of parameters, which can be easily verified by simulations. Nonetheless, such a choice is arbitrary and does not affect the analysis.

During the off-time of each diode, the circuit state is constrained to lie in the space defined by the zero value of the cor-

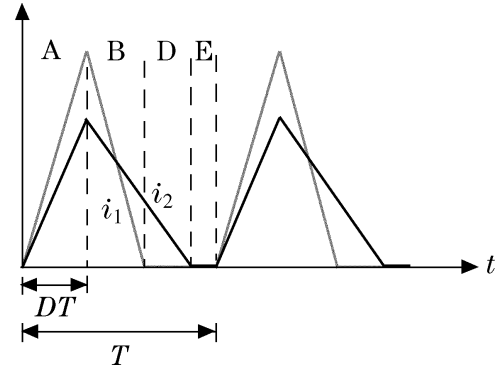

Fig. 3. Typical current waveforms of the single-stage PFC power supply. Both the boost and the buck stages operate in DCM.

responding current. Thus, $i_{1}$ can be ignored for State $\mathrm{D}$, and both $i_{1}$ and $i_{2}$ can be ignored for State E. Then, according to the circuit topology in each switch state, we can write the state equations as follows:

$$
\begin{cases}\dot{x}=A_{1} x+B e(t), & \text { for State A } \\ \dot{x}=A_{2} x+B e(t), & \text { for State B } \\ \dot{y}=A_{3} y, & \text { for State D } \\ \dot{v}=A_{4} v, & \text { for State E }\end{cases}
$$

where $e(t)$ is the input voltage, the state vectors are defined as $x=\left[\begin{array}{llll}i_{1} & v_{C} & i_{2} & v_{o}\end{array}\right]^{T}, y=\left[\begin{array}{lll}v_{C} & i_{2} & v_{o}\end{array}\right]^{T}$, and $v=\left[\begin{array}{ll}v_{C} & v_{o}\end{array}\right]^{T}$, and the system matrices for this converter are

$$
\begin{aligned}
A_{1}= & {\left[\begin{array}{cccc}
-\frac{r_{1}}{L_{1}} & 0 & 0 & 0 \\
0 & 0 & -\frac{1}{C_{1}} & 0 \\
0 & \frac{1}{L_{2}} & -\frac{r_{2}}{L_{2}} & -\frac{1}{L_{2}} \\
0 & 0 & \frac{1}{C_{2}} & -\frac{1}{C_{2} R}
\end{array}\right] } \\
A_{2}= & {\left[\begin{array}{cccc}
-\frac{r_{1}}{L_{1}} & -\frac{1}{L_{1}} & 0 & 0 \\
\frac{1}{C_{1}} & 0 & 0 & 0 \\
0 & 0 & -\frac{r_{2}}{L_{2}} & -\frac{1}{L_{2}} \\
0 & 0 & \frac{1}{C_{2}} & -\frac{1}{C_{2} R}
\end{array}\right] } \\
A_{3}= & {\left[\begin{array}{ccc}
0 & 0 & 0 \\
0 & -\frac{r_{2}}{L_{2}} & -\frac{1}{L_{2}} \\
0 & \frac{1}{C_{2}} & -\frac{1}{C_{2} R}
\end{array}\right] } \\
A_{4}= & {\left[\begin{array}{ll}
0 & 0 \\
0 & -\frac{1}{C_{2} R}
\end{array}\right] } \\
B= & {\left[\begin{array}{l}
\frac{1}{L_{1}} \\
0 \\
0 \\
0
\end{array}\right] }
\end{aligned}
$$



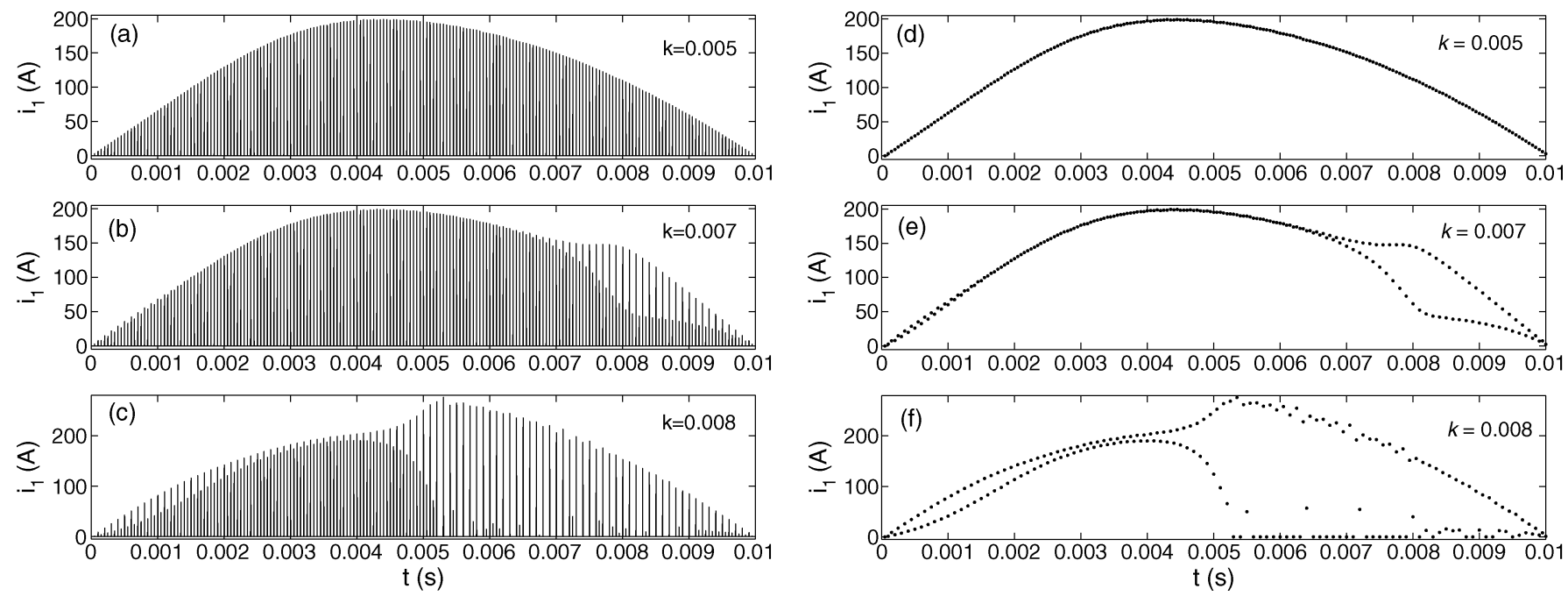

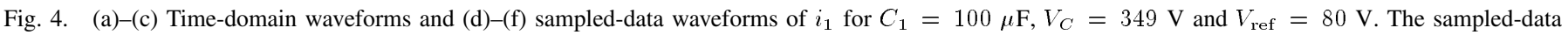
waveforms are obtained by sampling the peak values of the waveforms and hence indicate the instantaneous current stress.

TABLE I

COMPONENT VALUES USED IN SIMULATIONS

\begin{tabular}{ll}
\hline Circuit Components & Values \\
\hline Switching Period $T$ & $50 \mu \mathrm{s}$ \\
Input Voltage & $110 \mathrm{~V} \mathrm{rms}, 50 \mathrm{~Hz}$ \\
Inductance $L_{1}$, ESR $r_{1}$ & $2 \mu \mathrm{H}, 0.01 \Omega$ \\
Inductance $L_{2}$, ESR $r_{2}$ & $10 \mu \mathrm{H}, 0.01 \Omega$ \\
Capacitance $C_{1}$ & $10-100 \mu \mathrm{F}$ \\
Capacitance $C_{2}$ & $34 \mu \mathrm{F}$ \\
Load Resistance $R$ & $10 \Omega$ \\
\hline
\end{tabular}

where all component symbols are as defined in the circuit diagram shown in Fig. 2.

\section{COMPUTER SimUlation StUdy}

In this section, we begin with a series of computer simulations to identify possible bifurcation phenomena. Since we are primarily concerned with system stability in relation to the reference output voltage $V_{\text {ref }}$ and the feedback gain $k$, we will focus on variation of these two parameters. Our simulation is based on the state equations derived in the foregoing section. Essentially, for each set of parameter values, time-domain cycle-by-cycle waveforms are generated by solving the appropriate linear equation in a sub-interval of time, according to different switching states. After the transient period, we capture the steady-state time-domain waveforms. The circuit component values used are listed in Table I.

For a certain range of parameters, fast-scale instability may occur within a line cycle. Such instability manifests itself as a period-doubling bifurcation at the switching frequency.

\section{A. Simulation Results for Relatively Large Storage Capacitance}

We begin with a relatively large value of capacitance for $C_{1}$. We generally observe that fast-scale instability occurs near the two ends of a half line cycle, as shown in Fig. 4 for different values of the feedback gain $k$ for $C_{1}=100 \mu \mathrm{F}$. Fig. 4(a) shows the stable operation with $k=0.005$. Fig. 4(b) shows bifurcation in some intervals of the line cycle with $k=0.007$. Fig. 4(c) shows fast-scale instability in the whole line cycle with $k=0.008$. Specifically, period doublings at the switching period occurs at some phase angle of the line cycle and instability grows toward the zero-crossings of the line cycle. In order to observe the change in dynamical behavior clearly, we collect the sampled peak values for $i_{1}$ and $i_{2}$ during each switching period in the steady state, and the corresponding sampled values for $v_{C}$ and $v_{o}$. Fig. 4(d)-(f) shows the corresponding sampled-data waveforms of Fig. 4(a)-(c). Period-doubling bifurcations are clearly observed. Fig. 5 shows the corresponding phase portraits of the sampled input current $i_{1}$ and voltage $v_{C}$.

Of engineering importance is the existence (and location) of the critical bifurcation points along the line cycle as it affects the peak current value of the input current and hence the current stress on the switch. We denote the two critical points in terms of phase angle $\theta_{c 1}=\omega t_{1}$ and $\theta_{c 2}=\omega t_{2}$, where $\omega$ is the line angular frequency. Fig. 6 plots the two critical phase angles $\theta_{c 1}$ and $\theta_{c 2}$ as functions of the feedback gain $k$ and $V_{\text {ref. }}$. Here, we observe that the converter fails to maintain the expected stability operation in intervals corresponding to $\theta<\theta_{c 1}$ and $\theta>\theta_{c 2}$.

Furthermore, for a certain reference output voltage $V_{\text {ref }}$, there is a critical value of feedback gain $k_{c}$, above which the operation has regions of fast-scale instability. Likewise, for a given feedback gain $k$, there is a critical reference output voltage $V_{\text {ref }_{c}}$, below which the operation has regions of fast-scale instability. Consequently, these critical parameter values define a stability boundary dividing the parameter space of the feedback gain and the reference output voltage, as shown in Fig. 7.

\section{B. Simulation Results for Relatively Small Storage Capacitance}

We now examine the system when the capacitance $C_{1}$ is relatively small. We observe that, unlike in the case for large $C_{1}$, fast-scale instability grows in the middle of a half line cycle, 

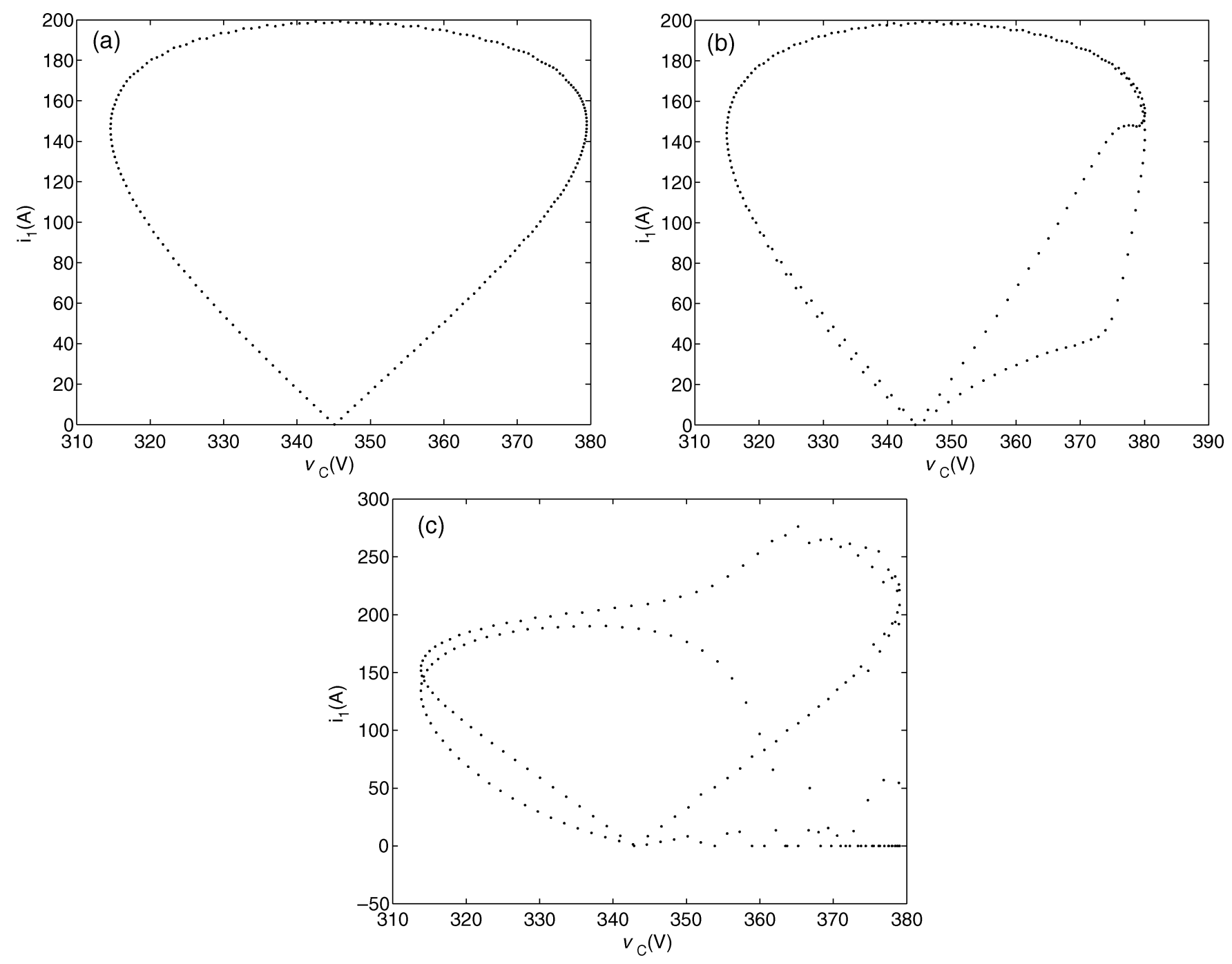

Fig. 5. Phase portraits of sampled $i_{1}$ versus $v_{C}$ for $C_{1}=100 \mu \mathrm{F}, V_{C}=349 \mathrm{~V}$ and $V_{\text {ref }}=80 \mathrm{~V}$.

i.e., the regions near the zero-crossings being fast-scale stable. Fig. 8 shows various scenarios with different values of the feedback gain $k$ for $C_{1}=10 \mu \mathrm{F}$. Fig. 8(a) shows fast-scale instability in some interval of the line cycle for $k=0.006$. Fig. 8(b) shows the fast-scale period-2 and period-4 (with respect to the switching period) operations in some interval of the line cycle for $k=0.007$. Fig. 8 (c) shows fast-scale instability in some intervals of the line cycle for $k=0.01$. Fig. 8(d)-(f) shows the corresponding sampled-data waveforms.

Similarly, we denote the two critical points in terms of phase angle as $\theta_{c 1}$ and $\theta_{c 2}$. Fig. 9 plots the two critical phase angles $\theta_{c 1}$ and $\theta_{c 2}$ as functions of the feedback gain $k$. Here, we observe that the converter fails to maintain the desired stable operation in the interval $\theta_{c 1}<\theta<\theta_{c 2}$.

\section{Effects of Storage Capacitance on Fast-Scale Instability}

Here, we observe two apparently different instability manifestations. Specifically, for relatively large storage capacitance, the growth of fast-scale instability takes place near zero-crossings of the line cycle, whereas for relatively small storage capacitance, such fast-scale instability occurs near the high inputvoltage regions of the line cycle. In this subsection, we present a detailed inspection of the effect of the size of the storage capacitance. Extensive simulations have been performed to track the values of the critical phase angles as $C_{1}$ increases. The results are shown in Fig. 10. There is no sudden jump of the critical phase angles as the storage capacitance varies. Instead, a continuous transition has taken place, as clearly illustrated in Fig. 10. This general transition, giving rise to the different locations of stability regions observed for large and small storage capacitance, are due to the phase shift of the storage capacitor voltage. Such a trend is general observed for different values of feedback gain $k$. Furthermore, the width of the stable region decreases as $k$ increases.

\section{TheORETICAL ANAlysis of FAST-SCALE BIFURCATION BEHAVIOR}

From the foregoing simulation study, we have identified period-doubling bifurcation in certain parameter ranges. In this section, we try to analyze the bifurcation in terms of suitable discrete-time model. Since the input voltage $e(t)$ is a rectified sine wave, whose frequency is much lower than the switching frequency (200 times less in this case), we can assume that the input voltage is a constant value equal to $e(n T)$ during a 

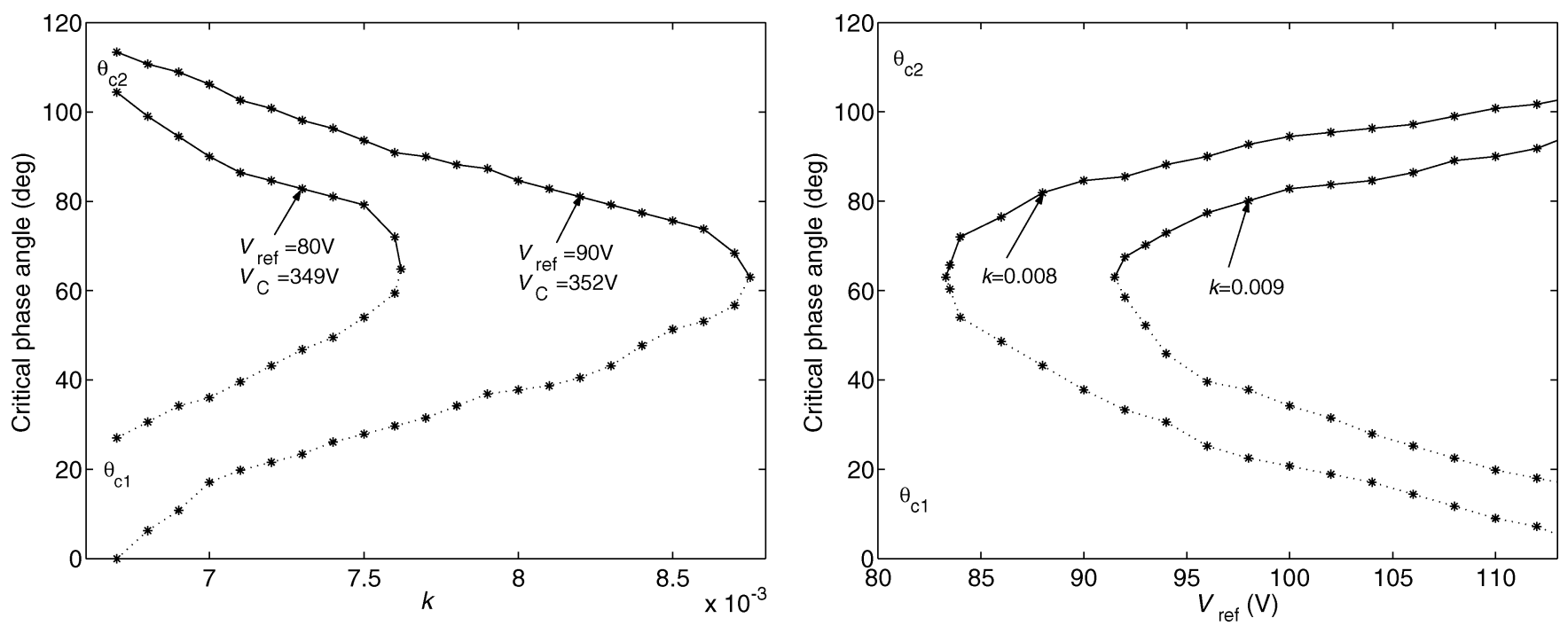

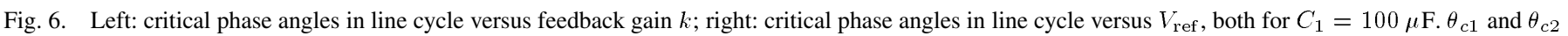
are the critical phase angles at which period-doubling bifurcation begins to occur. Fast-scale stable regions correspond to $\theta_{c 1}<\theta<\theta_{c 2}$.

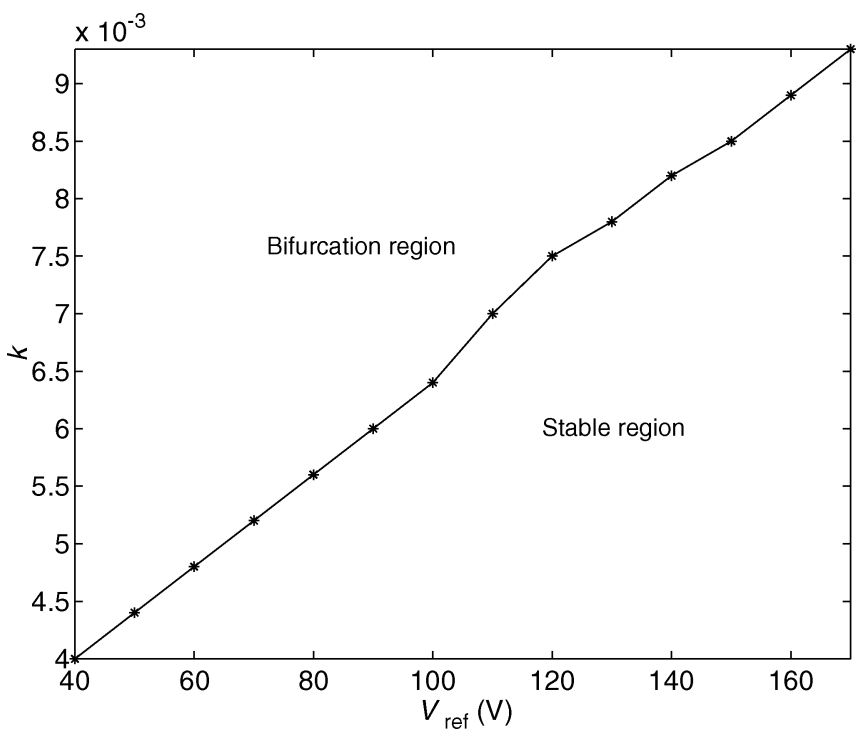

Fig. 7. Stability boundary in the parameter space of feedback gain versus output reference voltage for $C_{1}=100 \mu \mathrm{F}$.

switching period. The aim of the analytical study is to derive the discrete-time map describing the dynamics of the system. Then, by examining the Jacobian of this map, the stability can be assessed.

\section{A. Derivation of Discrete-Time Map}

Our purpose in this subsection is to derive a discrete-time map that describes the dynamics of the single-stage PFC converter operating in DCM. As mentioned before, due to the DCM, currents $i_{1}$ and $i_{2}$ do not act as state variables in this discrete-time model since $i_{1}\left(t_{n}\right)=0$ and $i_{2}\left(t_{n}\right)=0$ for all $n$. Thus, the iterative map we aim to find takes the following form

$$
v_{n+1}=f\left(v_{n}, d_{n}, h_{1, n}, h_{2, n}\right)
$$

where $v_{n}=v(n T)$ denotes the value at the beginning of the $n$th cycle, $d_{n}$ is the duty cycle in the $n$th cycle, $h_{1, n}$ is the fraction of the switching period during which $S_{1}$ is off and $i_{1}$ is nonzero, and $h_{2, n}$ is the fraction of the switching period during which $S_{2}$ is off and $i_{2}$ is nonzero.

It is necessary to correctly model the change in state-space dimension when the diodes switch off in order to obtain correct stability results. With $D_{1}$ switched off, the matrix projecting the four-dimensional state $x$ to the three-dimensional state $y$ is

$$
M=\left[\begin{array}{llll}
0 & 1 & 0 & 0 \\
0 & 0 & 1 & 0 \\
0 & 0 & 0 & 1
\end{array}\right]
$$

With $D_{2}$ switched off, the matrix projecting the three-dimensional state $y$ to the two-dimensional state $v$ is

$$
N=\left[\begin{array}{lll}
1 & 0 & 0 \\
0 & 0 & 1
\end{array}\right]
$$

With the switches turned on, the matrix augmenting the statespace dimension from two $(v)$ to four $(x)$ is

$$
Q=M^{T} N^{T}=\left[\begin{array}{ll}
0 & 0 \\
1 & 0 \\
0 & 0 \\
0 & 1
\end{array}\right]
$$

The state is unchanged in dimension and continuous at instants when the switches are turned off.

The state equations are given in (3) for different states. Here, without loss of generality, we omit state $\mathrm{C}$. Thus, there are four consecutive subintervals in one switching cycle.

1) For $n T \leq t<n T+d_{n} T, S_{1}$ and $S_{2}$ are on, $D_{1}$ and $D_{2}$ are off;

2) For $n T+d_{n} T \leq t<n T+d_{n} T+h_{1, n} T, S_{1}$ and $S_{2}$ are off, $D_{1}$ and $D_{2}$ are on;

3) For $n T+d_{n} T+h_{1, n} T \leq t<n T+d_{n} T+h_{2, n} T, S_{1}$ and $S_{2}$ are off, $D_{1}$ is off and $D_{2}$ is on;

4) For $n T+d_{n} T+h_{2, n} T \leq t<(n+1) T, S_{1}$ and $S_{2}$ are off, $D_{1}$ and $D_{2}$ are off. 

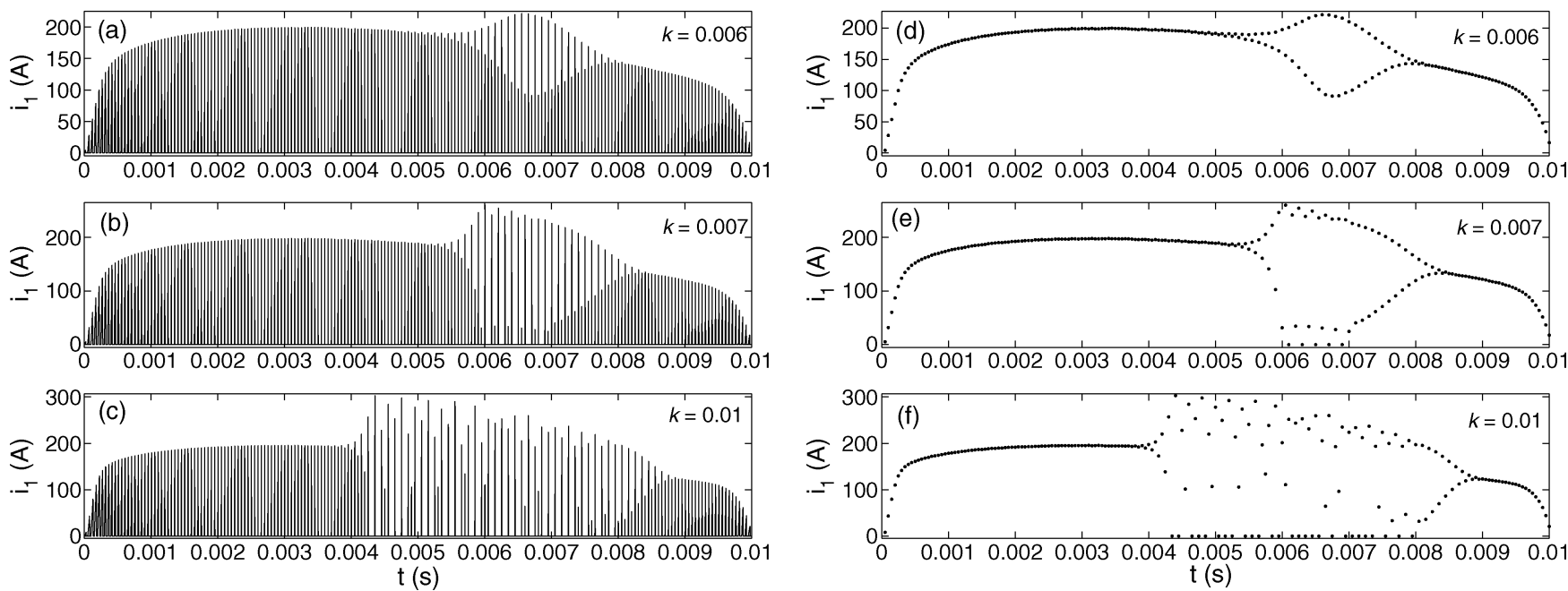

Fig. 8. (a)-(c) Time-domain waveforms and (d)-(f) sampled-data waveforms of $i_{1}$ for $C_{1}=10 \mu \mathrm{F}, V_{C}=321 \mathrm{~V}$ and $V_{\text {ref }}=90 \mathrm{~V}$.

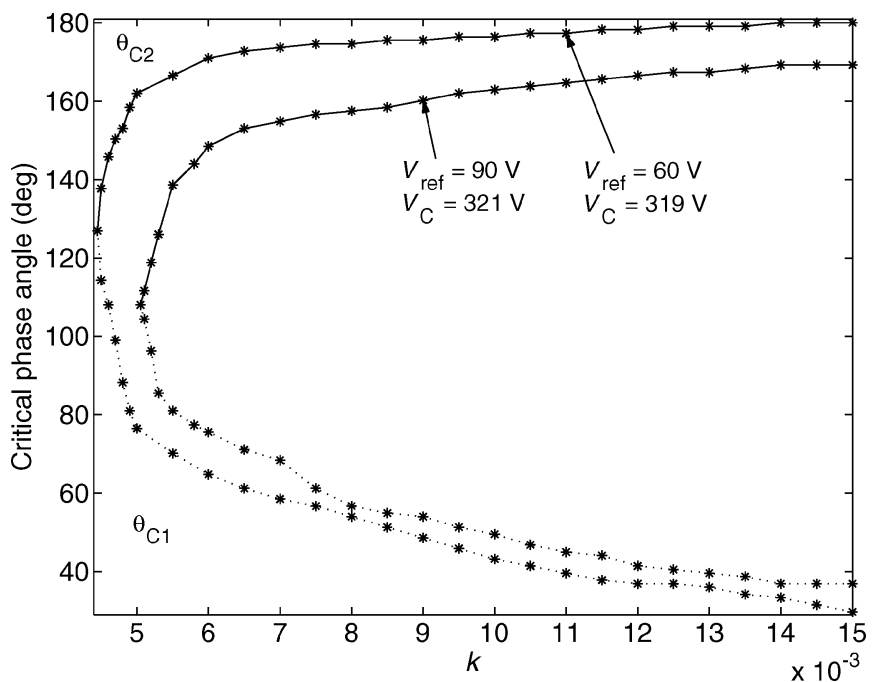

Fig. 9. Critical phase angles in line cycle versus feedback gain $k$ for $C_{1}=$ $10 \mu \mathrm{F}$. Fast-scale unstable regions correspond to $\theta_{c 1}<\theta<\theta_{c 2}$.

For the state equation in each subinterval, we can derive the solution, and by stacking up the solutions, $v_{n+1}$ can be expressed in terms of $v_{n}, d_{n}, h_{1, n}$, and $h_{2, n}$, as represented in (13), i.e.,

$$
\begin{aligned}
v_{n+1}= & \Phi_{4}\left(\bar{d}_{n} T\right) N \Phi_{3}\left(\bar{h}_{n} T\right) M \\
& \times\left\{\Phi_{2}\left(h_{1, n} T\right) \Phi_{1}\left(d_{n} T\right) M^{T} N^{T} v_{n}\right. \\
& \quad+\left[\Phi_{2}\left(h_{1, n} T\right)\left(\Phi_{1}\left(d_{n} T\right)-1\right) A_{1}^{-1}\right. \\
& \left.\left.\quad+\left(\Phi_{2}\left(h_{1, n} T\right)-1\right) A_{2}^{-1}\right] B e_{n}\right\}
\end{aligned}
$$

where $\bar{d}_{n}=1-h_{2, n}-d_{n}, \bar{h}_{n}=h_{2, n}-h_{1, n}, \mathbf{1}$ is the unit matrix, $e_{n}$ is the input voltage at the beginning of the $n$th cycle, and

$$
\Phi_{j}(\xi)=e^{A_{j} \xi}=\mathbf{1}+\sum_{n=1}^{\infty} \frac{1}{n !} A_{j}^{n} \xi^{n}, \quad \text { for } \quad j=1,2,3,4 .
$$

Our next step is to find the feedback equations that connect $d_{n}, h_{1, n}$ and $h_{2, n}$ to $v_{n}$. To find the defining equation for the duty cycle $d_{n}$, we first note that both switches are turned off when $v_{\text {con }}=V_{\text {ramp }}$. For brevity, we define

$$
\begin{aligned}
s\left(v_{n}, d_{n}\right)= & v_{\text {con }}-V_{\text {ramp }} \\
= & \left(V_{\text {offset }}+k V_{\text {ref }}-V_{L}\right)-\left(V_{U}-V_{L}\right) d_{n} \\
& -k v_{o}\left(d_{n} T\right)
\end{aligned}
$$

$$
\begin{gathered}
=\left(V_{\text {offset }}+k V_{\text {ref }}-V_{L}\right)-\left(V_{U}-V_{L}\right) d_{n} \\
-k_{1}^{T}\left[\Phi_{1}\left(d_{n} T\right) M^{T} N^{T} v_{n}\right. \\
\left.+\left(\Phi_{1}\left(d_{n} T\right)-1\right) A_{1}^{-1} B e_{n}\right]
\end{gathered}
$$

where $k_{1}^{T}=\left[\begin{array}{llll}0 & 0 & 0 & k\end{array}\right]$. Thus, $S_{1}$ and $S_{2}$ are turned off simultaneously when

$$
s\left(v_{n}, d_{n}\right)=0
$$

Also, $d_{n}, h_{1, n}$ and $h_{2, n}$ are related by enforcing continuity of the inductor currents at the switching instants [27], i.e.,

$$
\begin{aligned}
h_{1, n} & =\frac{e_{n}}{v_{C, n}-e_{n}} d_{n}=\frac{e_{n}}{k_{2}^{T} v_{n}-e_{n}} d_{n} \\
h_{2, n} & =\frac{v_{C, n}-v_{o, n}}{v_{o, n}} d_{n}=\frac{k_{2}^{T} v_{n}-k_{3}^{T} v_{n}}{k_{3}^{T} v_{n}} d_{n}
\end{aligned}
$$

where $k_{2}^{T}=\left[\begin{array}{ll}1 & 0\end{array}\right]$ and $k_{3}^{T}=\left[\begin{array}{ll}0 & 1\end{array}\right]$. Combining (16), (17), and (18) with (13) yields the discrete-time iterative map for the closed-loop system.

\section{B. Derivation of the Jacobian}

The Jacobian plays an important role in the study of dynamical systems. The essence of using a Jacobian in the analysis of the dynamical systems lies in the capture of the dynamics in the small neighborhood of an equilibrium point or orbit. We will make use of this conventional method to examine the bifurcation phenomena observed earlier in Section III.

Suppose the equilibrium point is given by $v(n T)=V_{Q}$. The Jacobian of the discrete-time map evaluated at the equilibrium point can be written as follows:

$$
\begin{aligned}
J\left(V_{Q}\right)=\frac{\partial f}{\partial v_{n}} & +\frac{\partial f}{\partial d_{n}} \frac{d d_{n}}{d v_{n}}+\frac{\partial f}{\partial h_{1, n}}\left(\frac{\partial h_{1, n}}{\partial v_{n}}+\frac{\partial h_{1, n}}{\partial d_{n}} \frac{d d_{n}}{d v_{n}}\right) \\
& +\left.\frac{\partial f}{\partial h_{2, n}}\left(\frac{\partial h_{2, n}}{\partial v_{n}}+\frac{\partial h_{2, n}}{\partial d_{n}} \frac{d d_{n}}{d v_{n}}\right)\right|_{v_{n}=V_{Q} .}
\end{aligned}
$$

Using (13), (16) and (18), we can find all the derivatives in (19). First, $\partial f / \partial v_{n}$ can be found from (13), i.e.,

$$
\frac{\partial f}{\partial v_{n}}=\Phi_{4}\left(\bar{d}_{n} T\right) N \Phi_{3}\left(\bar{h}_{n} T\right) M \Phi_{2}\left(h_{1, n} T\right) \Phi_{1}\left(d_{n} T\right) M^{T} N^{T} .
$$



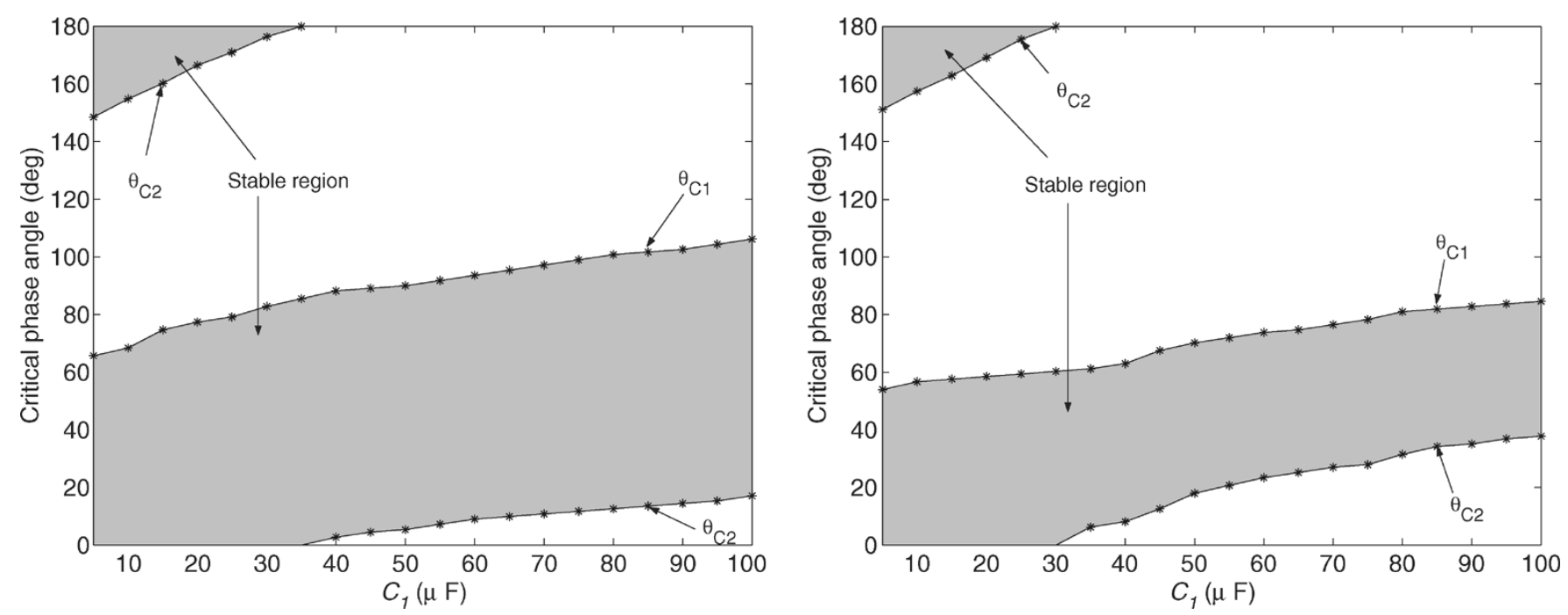

Fig. 10. Left: critical phase angles versus $C_{1}$, with $V_{\text {ref }}=90 \mathrm{~V}$ and $k=0.007$; right: critical phase angles versus $C_{1}$, with $V_{\text {ref }}=90 \mathrm{~V}$ and $k=0.008$.

Also, $\partial f / \partial d_{n}$ can be obtained as

$$
\begin{aligned}
\frac{\partial f}{\partial d_{n}}= & \Phi_{4}\left(\bar{d}_{n} T\right)\left(-A_{4} T\right) N \Phi_{3}\left(\bar{h}_{n} T\right) M \\
& \times\left[\Phi_{2}\left(h_{1, n} T\right) \Phi_{1}\left(d_{n} T\right) M^{T} N^{T} v_{n}\right. \\
& +\left(\Phi_{2}\left(h_{1, n} T\right)\left(\Phi_{1}\left(d_{n} T\right)-1\right) A_{1}^{-1}\right. \\
& \left.\left.\quad+\left(\Phi_{2}\left(h_{1, n} T\right)-1\right) A_{2}^{-1}\right) B e_{n}\right] \\
& +\Phi_{4}\left(\bar{d}_{n} T\right) N \Phi_{3}\left(\bar{h}_{n} T\right) M \\
& \times\left[\Phi_{2}\left(h_{1, n} T\right) \Phi_{1}\left(d_{n} T\right) A_{1} T M^{T} N^{T} v_{n}\right. \\
& \left.\quad+\Phi_{2}\left(h_{1, n} T\right) \Phi_{1}\left(d_{n} T\right) B e_{n}\right] \\
= & T A_{4} \Phi_{4}\left(\bar{d}_{n} T\right) N \Phi_{3}\left(\bar{h}_{n} T\right) M x\left(n T+d_{n} T+h_{1, n} T\right) \\
& +T \Phi_{4}\left(\bar{d}_{n} T\right) N \Phi_{3}\left(\bar{h}_{n} T\right) M \Phi_{2}\left(h_{1, n} T\right) \\
& \times \Phi_{1}\left(d_{n} T\right) \dot{x}(n T)_{+}
\end{aligned}
$$

where $\dot{x}(t)_{+}$denotes the right-hand side time derivative of state vector $x$ at time $t$. In (21), the first term corresponds to cascading the solutions of the state equations given in (3) and, therefore, equals $-T A_{4} v_{n+1}$. By applying the appropriate transition matrices and (10), (11), and

$$
M A_{2} M^{T}=A_{3} ; \quad M B=0 ; \quad N A_{3} N^{T}=A_{4}
$$

we can put the second term of (21) as

$$
\begin{aligned}
&\left.T \Phi_{4}\left(\bar{d}_{n} T\right) N \Phi_{3}\left(\bar{h}_{n} T\right) M \Phi_{2}\left(h_{1, n} T\right)\left[A_{1} x\left(n T+d_{n} T\right)+B e_{n}\right)\right] \\
&= T \Phi_{4}\left(\bar{d}_{n} T\right) N \Phi_{3}\left(\bar{h}_{n} T\right) M \Phi_{2}\left(h_{1, n} T\right) \\
& \times\left[\dot{x}\left(n T+d_{n} T\right)_{+}+\left(A_{1}-A_{2}\right) x\left(n T+d_{n} T\right)\right] \\
&= T \Phi_{4}\left(\bar{d}_{n} T\right) N \Phi_{3}\left(\bar{h}_{n} T\right) \\
& \quad \times {\left[\dot{y}\left(n T+d_{n} T+h_{1, n} T\right)_{+}\right.} \\
&\left.+M \Phi_{2}\left(h_{1, n} T\right)\left(A_{1}-A_{2}\right) x\left(n T+d_{n} T\right)\right] \\
&= T \Phi_{4}\left(\bar{d}_{n} T\right) N \\
& \times\left[A_{3} y\left(n T+d_{n} T+h_{2, n} T\right)\right. \\
&\left.\quad+\Phi_{3}\left(\bar{h}_{n} T\right) M \Phi_{2}\left(h_{1, n} T\right)\left(A_{1}-A_{2}\right) x\left(n T+d_{n} T\right)\right] \\
&= T \Phi_{4}\left(\bar{d}_{n} T\right) \\
& \times\left[N A_{3} N^{T} v\left(n T+d_{n} T+h_{2, n} T\right)\right. \\
&\left.+N \Phi_{3}\left(\bar{h}_{n} T\right) M \Phi_{2}\left(h_{1, n} T\right)\left(A_{1}-A_{2}\right) x\left(n T+d_{n} T\right)\right] \\
&= T A_{4} v_{n+1}+T \Phi_{4}\left(\bar{d}_{n} T\right) N \Phi_{3}\left(\bar{h}_{n} T\right) \\
& \times M \Phi_{2}\left(h_{1, n} T\right)\left(A_{1}-A_{2}\right) x\left(n T+d_{n} T\right) .
\end{aligned}
$$

Hence, $\partial f / \partial d_{n}$ can be expressed as

$$
\begin{aligned}
\frac{\partial f}{\partial d_{n}}=T \Phi_{4}\left(\bar{d}_{n} T\right) N \Phi_{3}\left(\bar{h}_{n} T\right) M \Phi_{2}\left(h_{1, n} T\right) \\
\times\left(A_{1}-A_{2}\right) x\left(n T+d_{n} T\right) .
\end{aligned}
$$

Likewise, we obtain

$$
\begin{aligned}
& \frac{\partial f}{\partial h_{1, n}}=\Phi_{4}\left(\bar{d}_{n} T\right) N \Phi_{3}\left(\bar{h}_{n} T\right)\left(-A_{3} T\right) M \\
& \times\left\{\Phi_{2}\left(h_{1, n} T\right) \Phi_{1}\left(d_{n} T\right) M^{T} N^{T} v_{n}\right. \\
& +\left[\Phi_{2}\left(h_{1, n} T\right)\left(\Phi_{1}\left(d_{n} T\right)-1\right) A_{1}^{-1}\right. \\
& \left.\left.+\left(\Phi_{2}\left(h_{1, n} T\right)-1\right) A_{2}^{-1}\right] B e_{n}\right\} \\
& +\Phi_{4}\left(\bar{d}_{n} T\right) N \Phi_{3}\left(\bar{h}_{n} T\right) M \\
& \times\left\{\Phi_{2}\left(h_{1, n} T\right) A_{2} T \Phi_{1}\left(d_{n} T\right) M^{T} N^{T} v_{n}\right. \\
& +\left[\Phi_{2}\left(h_{1, n} T\right) A_{2} T\left(\Phi_{1}\left(d_{n} T\right)-\mathbf{1}\right) A_{1}^{-1}\right. \\
& \left.\left.+\Phi_{2}\left(h_{1, n} T\right) T\right] B e_{n}\right\} \\
& =T \Phi_{4}\left(\bar{d}_{n} T\right) N \Phi_{3}\left(\bar{h}_{n} T\right) \\
& \times\left\{-A_{3} M x\left(n T+d_{n} T+h_{1, n} T\right)+M \Phi_{2}\left(h_{1, n} T\right)\right. \\
& \times\left[A _ { 2 } \left(\Phi_{1}\left(d_{n} T\right) M^{T} N^{T} v_{n}\right.\right. \\
& \left.\left.\left.+\left(\Phi_{1}\left(d_{n} T\right)-1\right) A_{1}^{-1} B e_{n}\right)+B e_{n}\right]\right\} \\
& =T \Phi_{4}\left(\bar{d}_{n} T\right) N \Phi_{3}\left(\bar{h}_{n} T\right) \\
& \times\left[-\dot{y}\left(n T+d_{n} T+h_{1, n} T\right)_{+}\right. \\
& \left.+M A_{2} M^{T} y\left(n T+d_{n} T+h_{1, n} T\right)\right] \\
& =0 .
\end{aligned}
$$

Furthermore

$$
\begin{aligned}
\frac{\partial f}{\partial h_{2, n}}= & T \Phi_{4}\left(\bar{d}_{n} T\right)\left(-A_{4} N+N A_{3}\right) \Phi_{3}\left(\bar{h}_{n} T\right) M \\
& \times\left\{\Phi_{2}\left(h_{1, n} T\right) \Phi_{1}\left(d_{n} T\right) M^{T} N^{T} v_{n}\right. \\
& \quad+\left[\Phi_{2}\left(h_{1, n} T\right)\left(\Phi_{1}\left(d_{n} T\right)-\mathbf{1}\right) A_{1}^{-1}\right. \\
& \left.\left.\quad+\left(\Phi_{2}\left(h_{1, n} T\right)-\mathbf{1}\right) A_{2}^{-1}\right] B e_{n}\right\} \\
= & T \Phi_{4}\left(\bar{d}_{n} T\right) N A_{3} k_{4} k_{4}^{T} y\left(n T+d_{n} T+h_{2, n} T\right) \\
= & 0
\end{aligned}
$$

since $-N^{T} N+1=k_{4} k_{4}^{T}, k_{4}^{T}=\left[\begin{array}{lll}0 & 1 & 0\end{array}\right]$, and the condition for diode $D_{2}$ to switch off is

$$
i_{2}\left(n T+d_{n} T+h_{2, n} T\right)=k_{4}^{T} y\left(n T+d_{n} T+h_{2, n} T\right)=0 .
$$



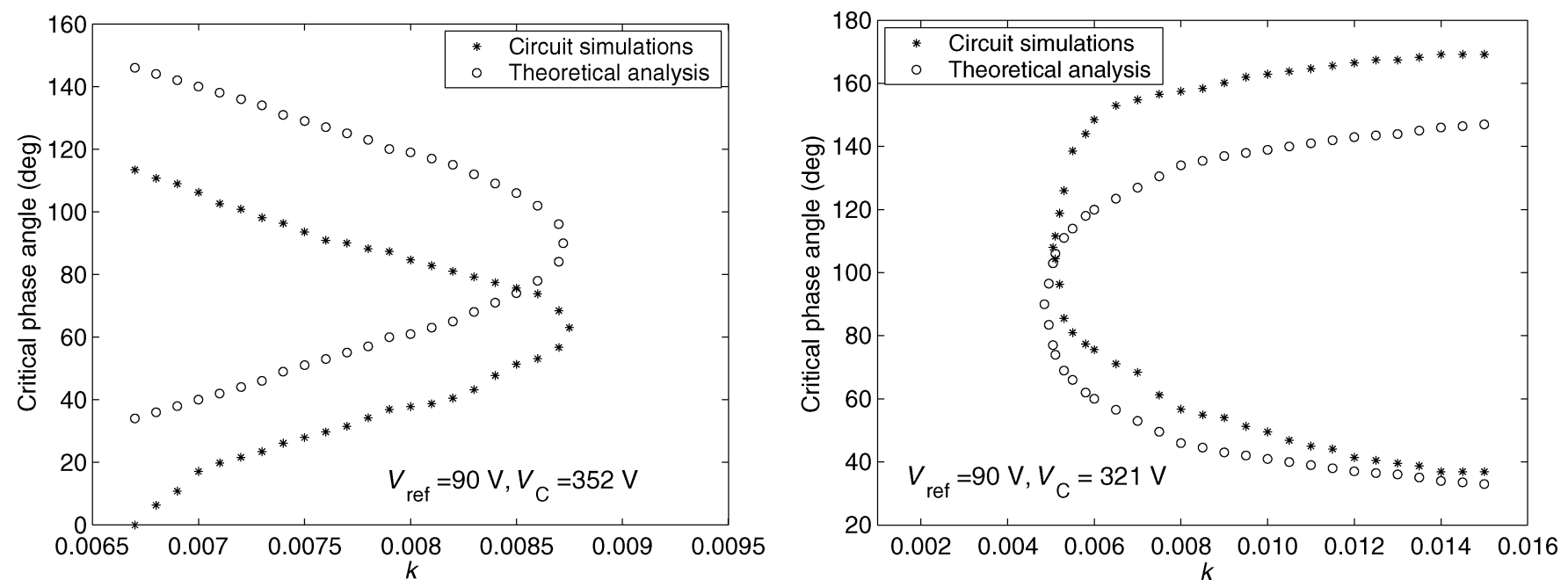

Fig. 11. Left: comparison of critical phase angles obtained from analysis and simulations for $C_{1}=100 \mu \mathrm{F}$; right: comparison of critical phase angles obtained from analysis and simulations for $C_{1}=10 \mu \mathrm{F}$. The difference between theoretical and simulation data can be attributed to the fact that analytical computation of the Jacobian assumes a steady-state operating point for each value of the input voltage, but such a steady state is never reached in the simulations in which time-varying input voltage has been used.

Now, from (25) and (26), the final state $v_{n+1}$ is first-order independent of the switch-off times of the diodes. Thus, $d d_{n} / d v_{n}$ is the last derivative needed to compute (19). From (15), we have

$$
\begin{aligned}
\frac{d d_{n}}{d v_{n}} & =-\left(\frac{\partial s}{\partial d_{n}}\right)^{-1} \frac{\partial s}{\partial v_{n}} \\
& =-\frac{k_{1}^{T} \Phi_{1}\left(d_{n} T\right) M^{T} N^{T}}{\left(V_{U}-V_{L}\right)+T k_{1}^{T} \Phi_{1}\left(d_{n} T\right)\left(A_{1} M^{T} N^{T} v_{n}+B e_{n}\right)} .
\end{aligned}
$$

Finally, substituting the derivatives obtained above in (19), the Jacobian is obtained as shown in (29) at the bottom of the page.

\section{Computation of Critical Phase Angles}

The Jacobian derived here can be used to evaluate the dynamics of the system. We study the loci of the characteristic multipliers, the aim being to identify possible bifurcation scenarios as the input voltage varies with time. To find the characteristic multipliers, we solve the following polynomial equation in $\lambda$ :

$$
\operatorname{det}\left[\lambda \mathbf{1}-J\left(V_{Q}\right)\right]=0 .
$$

In a line cycle, the input voltage is $\hat{e}|\sin \theta|$, where $0<\theta=$ $\omega t<\pi$. We track the movement of the characteristic multipliers as we vary the phase angle $\theta$. For a stable operation, all characteristic multipliers should stay inside the unit cycle. Any crossing from the interior of the unit cycle to the exterior indicates a bifurcation. For any set of parameters, we can compute the discrete-time map (13) and the corresponding Jacobian given in (29) for every $V_{Q}=v(n T)$, and record the values of $\theta=\omega t_{n}$ when one of the characteristic multipliers of the Jacobian reaches -1 . These are the critical phase angles at which period-doubling occurs. A comparison of these results with those obtained by computer simulations is shown in Fig. 11. The theoretical results generally match with the simulations. The discrepancies can be attributed to the fact that the computation of the Jacobian assumes a steady-state operating point for each value of the input voltage, but such a steady-state is never reached in the circuit simulations in which actual timevarying input voltage has been used. Thus, the simulation data should more realistically locate the stability boundaries, while the theoretical results produced from the analytical expressions provide quick and reasonably close numerical estimates.

\section{CONCLUSION}

Power factor correction has become an important design consideration for switching power supplies. For low-power applications (below $200 \mathrm{~W}$ ), a cost effective solution is to use a singlestage design in which the PFC stage is integrated with the dc-dc power stage. The specific solution proposed by Redl et al. [1] has proven to be versatile for low-power applications in terms of ease of control and containment of voltage stresses. Such a design utilizes DCM of operation to simplify the control and to maintain a fixed (load independent) voltage stress in the storage capacitor. In this paper we have performed a detailed study of the fast-scale bifurcation behavior of this converter and we have investigated into the effects of various parameters on the stability of the system. Such fast-scale stability problems are important as they affect the peak current stresses imposed on the switching devices. The results obtained here can be used to facilitate parameter selection for guaranteeing stable operation.

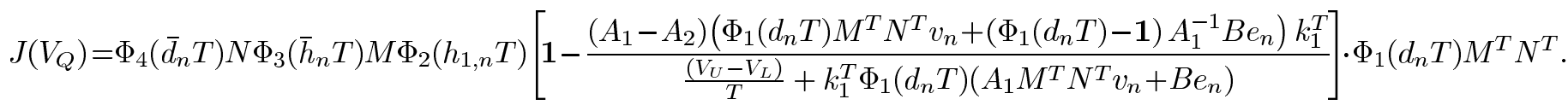




\section{ACKNOWLEDGMENT}

The authorss wish to thank one of the reviewers for pointing out an error in the derivation of the Jacobians in the first version of this paper.

\section{REFERENCES}

[1] R. Redl, L. Balogh, and N. O. Sokal, "A new family of single-stage isolated power-factor correctors with fast regulation of the output voltage," in IEEE PESC Rec., 1994, pp. 1137-1144.

[2] R. Redl, "Power factor correction in a single-stage switching-mode power supplies-an overview," Int. J. Electron., vol. 77, no. 5, pp. 555-582, 1994.

[3] C. K. Tse, "Circuit theory of power factor correction in switching converters," Int. J. Circuit Theory Appl., vol. 31, no. 1, pp. 157-198, Jan. 2003.

[4] K. W. Siu, Y. S. Lee, and C. K. Tse, "Analysis and experimental evaluation of single-switch fast response switching regulators with unity power factor," IEEE Trans. Ind. Appl., vol. 33, no. 5, pp. 1260-1266, Sep. 1997.

[5] M. H. L. Chow, K. W. Siu, C. K. Tse, and Y. S. Lee, "A novel method for elimination of line current harmonics in single-stage PFC switching regulators," IEEE Trans. Power Electron., vol. 13, no. 1, pp. 75-83, Jan. 1998.

[6] M. H. L. Chow, Y. S. Lee, and C. K. Tse, "Single-stage single-switch PFC regulator with unity power factor, fast transient response and low voltage stress," IEEE Trans. Power Electron., vol. 15, no. 1, pp. 156-163, Jan. 2000.

[7] J. H. B. Deane and D. C. Hamill, "Instability, subharmonics and chaos in power electronics circuits," IEEE Trans. Power Electron., vol. 5, no. 3, pp. 260-268, Oct. 1990.

[8] C. K. Tse, "Flip bifurcation and chaos in three-state boost switching regulators," IEEE Trans. Circuits Syst. I, Fundam. Theory, vol. 41, no. 1, pp. 16-23, Jan. 1994.

[9] S. Banerjee and K. Chakrabarty, "Nonlinear modeling and bifurcations in the boost converter," IEEE Trans. Power Electron., vol. 13, no. 2, pp. 252-260, Mar. 1998.

[10] E. Fossas and G. Olivar, "Study of chaos in the buck converter," IEEE Trans. Circuits Syst. I, Fundam. Theory Appl., vol. 43, no. 1, pp. 13-25, Jan. 1996.

[11] M. di Bernardo and F. Vasca, "Discrete-time maps for the analysis of bifurcations and chaos in dc/dc converters," IEEE Trans. Circuits Syst. I, Fundam. Theory Appl., vol. 47, no. 2, pp. 130-143, Feb. 2000.

[12] S. K. Mazumder, A. H. Nayfeh, and D. Boroyevich, "Theoretical and experimental investigation of the fast- and slow-scale instabilities of a dc/dc converter," IEEE Trans. Power Electron., vol. 16, no. 2, pp. 201-216, Mar. 2001.

[13] J. H. B. Deane, "Chaos in a current-mode controlled boost converter," IEEE Trans. Circuits Syst. I, Fundam. Theory Appl., vol. 39, no. 8, pp. 680-683, Aug. 1992.

[14] J. L. R. Marrero, J. M. Fort, and G. Verghese, "Analysis of the chaotic regime for dc-dc converters under current-mode control," in IEEE Power Electron. Specialists Conf. Rec., 1996, pp. 1477-1483.

[15] W. C. Y. Chan and C. K. Tse, "Study of bifurcation in current-programmed dc-dc boost converters: from quasi-periodicity to period-doubling," IEEE Trans. Circuits Syst. I, Fundam. Theory Appl., vol. 44, no. 12, pp. 1129-1142, Dec. 1997.

[16] I. Dobson, "Stability of ideal thyristor and diode switching circuits," IEEE Trans. Circuits Syst. I, Fundam. Theory Appl., vol. 42, no. 9, pp. 517-529, Sep. 1995.

[17] S. Jalali, I. Dobson, R. H. Lasseter, and G. Venkataramanan, "Switching time bifurcation in a thyristor controlled reactor," IEEE Trans. Circuits Syst. I, Fundam. Theory Appl., vol. 43, no. 3, pp. 209-218, Mar. 1996.

[18] C. K. Tse, Y. M. Lai, and H. H. C. Iu, "Hopf bifurcation and chaos in a free-running current-controlled Ćuk switching converter," IEEE Trans. Circuits Syst. I, Fundam. Theory Appl., vol. 47, no. 4, pp. 448-457, Apr. 2000.

[19] H. H. C. Iu and C. K. Tse, "Bifurcation behavior in parallel-connected buck converters," IEEE Trans. Circuits Syst. I, Fundam. Theory Appl., vol. 48, no. 2, pp. 233-240, Feb. 2001.

[20] H. H. C. Iu, C. K. Tse, V. Pjevalica, and Y. M. Lai, "Bifurcation behavior in parallel-connected boost converters," Int. J. Circuit Theory Appl., vol. 29, no. 3, pp. 281-298, 2001.
[21] T. Kousaka, T. Ueta, and H. Kawakami, "Bifurcation in switched nonlinear dynamical systems," IEEE Trans. Circuits Syst. II, Analog Digit. Signal Process., vol. 46, no. 7, pp. 878-885, Jul. 1999.

[22] Y. Ma, H. Kawakami, and C. K. Tse, "Bifurcation analysis of switched dynamical systems with periodically moving borders," IEEE Trans. Circuits Syst. I, Reg. Papers, vol. 51, no. 6, pp. 1184-1193, Jun. 2004.

[23] M. Orabi and T. Ninomiya, "Nonlinear dynamics of power-factor-correction converter," IEEE Trans. Ind. Electron., vol. 50, no. 6, pp. 1116-1125, Dec. 2003.

[24] H. H. C. Iu, Y. Zhou, and C. K. Tse, "Fast-scale instability in a PFC boost converter under average current mode control," Int. J. Circuit Theory Appl., vol. 31, no. 6, pp. 611-624, 2003.

[25] O. Dranga, C. K. Tse, H. H. C. Iu, and I. Nagy, "Bifurcation behavior of a power-factor-correction boost converter," Int. J. Bifur. Chaos, vol. 13, no. 10, pp. 3107-3114, 2003.

[26] S. Banerjee and G. Verghese, Eds., Nonlinear Phenomena in Power Electronics. New York: IEEE, 2001.

[27] C. K. Tse, Complex Behavior of Switching Power Converters. Boca Raton, FL: CRC, 2003.

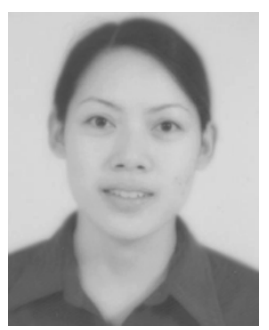

Xiaoqun Wu was born in Hubei, China, in 1978. She received the B.Sc. degree in applied mathematics and the $\mathrm{Ph} . \mathrm{D}$. degree in computational mathematics from Wuhan University, Hubei, China, in 2000 and 2005, respectively.

From February 2004 to March 2005, she was a Research Assistant with the Department of Electronic and Information Engineering, Hong Kong Polytechnic University, Hong Kong. Since May 2005, she has been a lecturer with the School of Mathematics and Statistics, Wuhan University, Hubei, China. She is presently a Research Associate with Hong Kong Polytechnic University. Her research interests include nonlinear dynamics, chaos control and synchronization, and bifurcation analysis in power electronics.

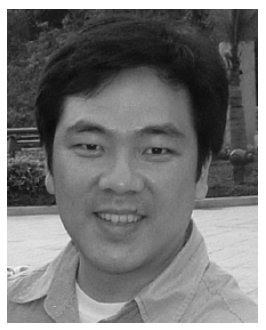

Chi K. Tse (M'90-SM'97-F'06) received the B.Eng. (Hons.) degree with first class honors in electrical engineering and the Ph.D. degree from the University of Melbourne, Australia, in 1987 and 1991, respectively.

$\mathrm{He}$ is presently a Chair Professor with Hong Kong Polytechnic University, Hong Kong, and his research interests include chaotic dynamics and power electronics. He is the author of Linear Circuit Analysis (London, U.K.: Addison-Wesley, 1998) and Complex Behavior of Switching Power Converters (Boca Raton: CRC Press, 2003), coauthor of Chaos-Based Digital Communication Systems (Heidelberg, Germany: Springer-Verlag, 2003) and Signal Reconstruction With Applications to Chaos-Based Communications (Beijing, China: Tsinghua University Press, 2005), and co-holder of a U.S. patent.

From 1999 to 2001,he served as an Associate Editor for the IEEE TRANSACTIONS ON CIRCUITS AND SYSTEMS-I: FUNDAMENTAL THEORY AND APPLICATIONS, and since 1999 he has been an Associate Editor for the IEEE TRANSACTIONS ON POWER ELECTRONICS. In 2005, he was appointed as an IEEE Distinguished Lecturer. He currently also serves as an Associate Editor of the International Journal of Systems Science, a Guest Associate Editor of the IEICE Transactions on Fundamentals of Electronics, Communications and Computers, and a Guest Editor of Circuits, Systems and Signal Processing. Since 2002, he has been appointed as Guest Professor by the Southwest China Normal University, Chongqing, China. In 1987, he was awarded the L.R. East Prize by the Institution of Engineers, Australia. He won the IEEE TRANSACTIONS ON POWER Electronics Prize Paper Award for 2001 and the International Journal of Circuit Theory and Applications Best Paper Award for 2003. While with Hong Kong Polytechnic University, he received the President's Award for Achievement in Research twice, the Faculty's Best Researcher Award, the Research Grant Achievement Award and a few other teaching awards. 


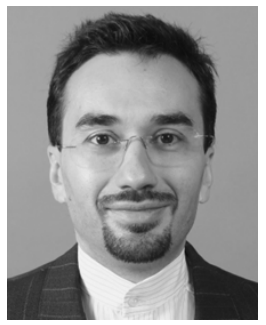

Octavian Dranga was born in Romania in 1971. He received the B.Sc., M.Sc., and Ph.D. degrees in control engineering from the "Politechnica" University of Timiooara, Timiooara, Romania in 1995, 1996, and 2001, respectively. the

He was an Assistant Lecturer with the "Politehnica" University of Timiooara from 1996 to 1998. During 1998-2001, he was a Researcher at Budapest University of Technology and Economics, Budapest, Hungary. He held postdoctoral research positions with Hong Kong Polytechnic University (2001-2002), University of Hull, Hull, U.K. (2003), and Utsunomiya University, Tochigi, Japan (2003-2004). He is currently with the Hong Kong Polytechnic University as a Research Fellow. His research interest is in the study of nonlinear phenomena in power electronics. He has authored 50 technical publications.

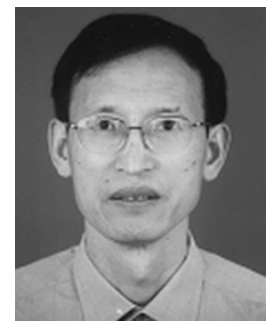

Junan Lu received the B.Sc. degree in geophysics from Peking University, China, and the M.Sc. degree in applied mathematics from Wuhan University, China, in 1968 and 1982, respectively.

$\mathrm{He}$ is currently a Professor with the School of Mathematics and Statistics, Wuhan University, Hubei, China. His research interests include nonlinear systems, chaos control and synchronization, complex networks, and scientific and engineering computing. He is the coauthor of An Introduction to Chaotic Dynamics, Analysis of Chaotic Time Series and Its Applications, and Matlab Solution of Partial Differential Equations. He has published more than 70 journal papers. 